\title{
Social prescribing
}

\section{Hans Stalder}

Prof. Dr méd., membre de la rédaction

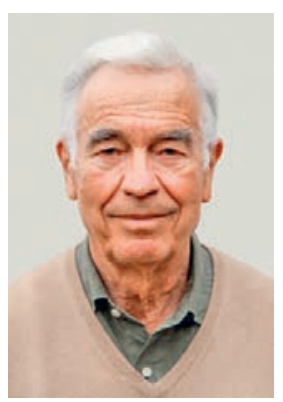

The number of annual deaths attributable to low social support was similar to the number from lung cancer [1].

La définition de la santé de l'OMS - «un état de complet bien-être physique, mental et social»-, même si elle est aujourd'hui mise en question, avait l'avantage de mettre sur un pied d'égalité le bien-être social et les paramètres physiques ainsi que psychiques. Si pour ces deux derniers aspects nous possédons des outils diagnostiques et des traitements bien établis, ce n'est pas le cas pour les déterminants sociaux. Nous savons dépister les lipides, mesurer la tension artérielle ou encore découvrir une dépression. La façon d'approcher, d'investiguer ou de traiter les facteurs de risque sociaux reste peu investiguée, malgré le fait qu'il soit reconnu depuis longtemps qu'ils sont beaucoup plus importants que les facteurs biologiques [2] - et la Suisse ne fait pas exception [3].

Nous savons par exemple que les lacunes scolaires et la précarité jouent un rôle néfaste sur la santé, mais nous n'avons pas défini un seuil pathologique, comme cela existe pour le cholestérol ou la tension artérielle. D'autres facteurs sont encore plus difficiles à cerner et à quantifier, comme le lieu de vie, la pollution ou le bruit. Toujours est-il que différents questionnaires ont été développés pour établir un profil social pour chaque patient [4].

Si les facteurs socio-économiques sont si importants nous devrions aussi être capables de les améliorer. Ceci est bien sûr d'abord une affaire de politique de santé publique: diminuer la pauvreté et les discriminations, promouvoir l'accès à la santé, améliorer la formation dans le domaine de la santé, etc. Mais n'est-ce pas aussi la tâche de chaque médecin de se soucier des déterminants sociaux de ses patients et de les améliorer? C'est l'idée du social prescribing [5].

Social prescribing est défini comme "a mechanism for linking patients with non-medical sources of support within the community" [5]. Le service national de santé anglais (NHS) en fait une priorité [6]. Etant donné que chaque médecin généraliste n'est pas nécessairement spécialiste dans ce domaine, des link workers ont été introduits. Spécialistes du secteur social, ces link workers correspondraient chez nous à des travailleurs sociaux. Les interventions peuvent être multiples et variées: l'éducation thérapeutique bien connue chez nous en est une, mais d'autres activités en font partie, comme aider à payer les factures, se préoccuper des dettes, enlever des tapis qui font trébucher, trouver des activités sociales pour des personnes isolées, promouvoir l'activité physique, donner des conseils juridiques, organiser un cours de langue. Nombre de ces activités sont peu coûteuses et certaines sont accomplies par des bénévoles. Elles sont adaptées individuellement à chaque patient qui en a la nécessité: il s'agit donc d'une médecine personnalisée, non pour trouver le meilleur médicament, mais pour dépister et combler des déficits sociaux.

L'idée est saisissante. Comme pour la médecine personnalisée biologique, nous sommes au tout début d'un développement prometteur. Pour le moment, les quelques études et méta-analyses sur le social prescribing suggèrent, sans prouver définitivement, l'efficacité de telles interventions [5, 7]. Le NHS y investit beaucoup d'argent: il prévoit qu'à l'avenir presqu'un million de patients pourraient en profiter en Angleterre. On observe des mouvements similaires dans d'autres pays [6]. Cela doit nous rendre attentifs à l'évolution de ce nouveau secteur thérapeutique et, pourquoi pas, nous inciter à participer à son développement et à son évaluation. D’ores et déjà, il faut prévoir qu'une assistante sociale fasse partie de nos équipes interprofessionnelles!

\section{Références}

1 Daniel H, Bornstein SS, Kane GC; Health and Public Policy Committee of the American College of Physicians. Addressing Social Determinants to Improve Patient Care and Promote Health Equity: An American College of Physicians Position Paper. Ann Intern Med. 2018;168:577-8.

2 Marmot MG, Rose G, Shipley M, Hamilton PJ. Employment grade and coronary heart disease in British civil servants. J Epidemiol Community Health.1978;32:244-9.

3 https://www.bfs.admin.ch/bfs/fr/home/statistiques/sante/ determinants/situation-sociale.htm

4 LaForge K, Gold R, Cottrell E, Bunce AE, Proser M, et al. How 6 organizations developed tools and processes for social determinants of health screening in primary care: An overview. J Ambul Care Manage. 2018;41:2-14.

5 Chatterjee HJ, CamicPM, Lockyerb B, Thomson LJM. Non-clinical community interventions of social prescribing schemes: a systematised review. Arts \& Health. 2018;10:97-123.

6 Drinkwater C, Wildman J, Moffatt S. Social prescribing. BMJ. 2019;364:1285-9.

7 Gottlieb LM, Wing H, Adler NE. A systematic review of interventions on patients' social and economic needs. Am J Prev Med. 2017;53:719-29. 